\title{
Human Papillomavirus 11 Antibody Measurement
}

National Cancer Institute

\section{Source}

National Cancer Institute. Human Papillomavirus 11 Antibody Measurement. NCI

Thesaurus. Code 127618.

The determination of the amount of human papillomavirus 11 antiboy present in a sample. 